Results Between January and December 2010, 227 MSM received STI services in the VICITS clinics in Tegucigalpa, San Pedro Sula and La Ceiba. Patients' mean age was 27.4 years. Overall 60.8\% (135/222) reported alcohol use and $34.7 \%$ (76/219) reported drug use during the last year. An STI in the previous 12 months was reported by $37.6 \%$ $(83 / 221)$ and $13.6 \%(30 / 219)$ reported engaging in sex work. Out of those patients who reported engaging in sex work, $86.5 \%(94 / 109)$ used a condom during their most recent sexual encounter, $88.3 \%$ (147) 166) reported using a condom with casual partners and $64.5 \%$ (74/ $115)$ with stable partners. Overall, $12.3 \%(10 / 81)$ were infected with HIV, $10.3 \%(13 / 126)$ had a confirmed syphilis infection, 3.6\% (5/138) were infected with chlamydia and gonorrhoea.

Conclusions Results from the first year of STI clinics for MSM evidence high HIV and syphilis prevalence rates. Behaviour change interventions targeting this community should focus on increasing condom use. Efforts should be made to increase the number of MSM attending specialised services.

\section{P1-S2.43 BEHAVIOURAL AND BIOLOGICAL SURVEILLANCE AMONG MAN WHO HAVE SEX WITH MAN USING RESPONDENT- DRIVEN SAMPLING METHODOLOGY IN MANAUS, AMAZON, BRAZIL}

\section{doi:10.1136/sextrans-2011-050108.100}

${ }^{1} \mathrm{~A} S$ Benzaken, ${ }^{2} \mathrm{E}$ G Galban, ${ }^{3} \mathrm{~N}$ S Benzaken, ${ }^{4} \mathrm{C} \mathrm{K},{ }^{5} \mathrm{~A}$ Pinho, ${ }^{6} \mathrm{M}$ Mello, ${ }^{1} \mathrm{~F}$ Vasquez, ${ }^{1} \mathrm{C}$ A Barros, ${ }^{7} \mathrm{~L}$ R Franco Sansigolo Kerr. ${ }^{1}$ Alfredo da Matta Foundation, Manaus, Brazil; ${ }^{2}$ Instituto Nacional de Gastroenterologia, Habana, Cuba; ${ }^{3}$ Nilton Lins University, Brazil; ${ }^{4}$ Tulane University, School of Public Health and Tropical Medicine, New Orleans, USA; ${ }^{5}$ Escola Nacional de Saùde Publica, Brazil; ${ }^{6}$ CDC, Brazil, Brazil; ${ }^{7}$ Uversidade Federal do Ceará, Brazil

Background There is a renewed interest in including men who have sex with men (MSM) in global HIV surveillance. A multicenter study of 10 Brazilian cities was designed to generate a national-level estimate the prevalence of HIV and syphilis among MSM. This abstracts presents the results of one of the participating cities.

Objectives To establish a baseline among MSM in Manaus to continuously monitor the prevalence of HIV and syphilis infection, level of knowledge about STI/HIV/AIDS, prevailing sexual attitudes and practices, and frequency of acts of discrimination against MSM. Methodology A cross-sectional study of 824 MSM (>18 years old) was conducted from October to May 2008 using respondent-driven sampling (RDS)- a chain-link and snowball sampling method that generates probability-based samples and is systematic, so it can be used to develop comparable surveillance data.

Results The weighted prevalence obtained with the use of Respondent-Driven Sampling Analysis Tool (RDSAT) software were $6.8 \%$ (CI 4.9 to 9.8) for HIV and 4.0\% (CI 2.6 to 5.8) for syphilis. A number of participants reported sex with both men and women (47.8\% of HIV positive and $56.1 \%$ of syphilis cases), while $20 \%$ of HIV positive and $30 \%$ of syphilis cases classified themselves as gay or homosexual. A previous infection of syphilis was the main predictive variables associated with HIV. The average age of first intercourse was 13.25 (with a range 5-23). This pattern of early sex suggests abuse. Fifty four point five per cent of participants had their first sexual intercourse with men, $42.6 \%$ with women, and $2.2 \%$ with transvestites. Participants also reported-41\% (CI 37 to 47) used condoms in their first relationship, 73.1\% (CI 69.7 to 77.2 ) had at least one casual sex partner during the previous 12 months, $31.6 \%$ (CI 27.7 to 35.9 ) used condoms consistently with casual relationships if they were with men and $15.5 \%$ (CI 11.9 to 18.9) with a woman. Nineteen per cent of MSM said they had been the target of direct physical aggression due to their sexual identity, 10\% reported being forced to have sex against their will, and over $30 \%$ reported being humiliated or disrespected because of their sexual orientation.
Conclusions The prevalence of HIV and syphilis infection in MSM in the city of Manaus is relatively high. There is substantial discrimination against MSM. The need of specific public policy and awareness campaigns for this vulnerable group is urgent.

\section{P1-S2.44 TRENDS IN STI IN MSM VISITING DUTCH STI CENTRES; DOES AGE MATTER?}

doi:10.1136/sextrans-2011-050108.101

${ }^{1} \mathrm{~F}$ Koedijk, ${ }^{2} \mathrm{P}$ van Beek, ${ }^{2} \mathrm{~K}$ Koops, ${ }^{1,3} \mathrm{M}$ A B van der Sande, ${ }^{4}$ On behalf of the Dutch STI centres. ${ }^{1}$ National Institute for Public Health and the Environment, Bilthoven, Netherlands; ${ }^{2}$ Schorer, Netherlands' institute for homosexuality, health and well-being, Netherlands; ${ }^{3}$ Academic Medical Centre Utrecht, University of Utrecht, Utrecht, the Netherlands, Netherlands; ${ }^{4} A$ v Daal (East), P v Leeuwen (North-Holland Flevoland), F de Groot (North), A Niekamp (Limburg), M Langevoort (Utrecht), A v Camerijk (South Holland North),J vd Sande (ZeeBRa),E vd Veen (South-Holland South), Netherlands

Objectives This study examined the distribution of selected sexually transmitted infections (STI) in men and men having sex with men (MSM) attending STI clinics in the Netherlands, separately for young ( $\geq 25$ years) and older ( $>25$ years) MSM.

Methods Data from 2004 up to June 2010 from the Dutch national surveillance in the STI centres were used to characterise trends in positivity rate in STI (at least one of the following-chlamydia, gonorrhoea, syphilis or HIV). Logistic regression was used to identify factors associated with STI positivity in both groups.

Results Older MSM tested more often positive for STI than younger MSM-21.5\% vs $18.4 \%$, respectively $(p<0.05)$. However, in older MSM there was a significant decreasing time trend in STI positivity (from 23\% in 2004 to 19\% in 2010, p < 0.05, abstract P1-S2.44 figure 1 ), while in young MSM the STI positivity rate remained stable over time. In multivariate analyses for young MSM, non-Dutch MSM tested significantly more often positive (OR $1.4,95 \%$ CI 1.3 to 1.6), as did those with a previous STI (OR 1.9, 95\% CI 1.6 to 2.1 ), known HIV positives (OR 3.1, 95\% CI 2.3 to 4.2), sex workers (OR 1.2, 95\% CI 1.1 to 1.6) and those with a low socio-economic status (OR 1.5, $95 \%$ CI 1.2 to 1.9). In older MSM, also non-Dutch MSM tested significantly more often positive (OR $1.2,95 \%$ CI 1.1 to 1.2 ), as did those with a previous STI (OR 1.6, 95\% CI 1.5 to 1.7), known HIV positives (OR 1.9, 95\% CI 1.8 to 2.1 ) and those with a low socioeconomic status (OR 1.2, 95\% CI 1.1 to 1.3). In contrast with young MSM, older MSM working as sex workers were at significantly lower risk (OR $0.7,95 \%$ CI 0.6 to 0.9 ) for testing positive for any STI.

Conclusions Young MSM are a specific group within total group of MSM, with some risk factors differencing from older MSM. While trends in positivity are decreasing over time in older MSM, they remain stable and high in young MSM. Therefore special attention needs to be paid towards counselling and reaching (specific groups of) young MSM, since they are at high risk for STI acquisition and transmission.

\section{P1-S2.45 CLUSTERS OFNEISSERIA GONORRHOEAE INFECTED PATIENTS ARE NOT LINKED TO HIV INFECTION WITHIN THE HOMOSEXUAL POPULATION IN AMSTERDAM, THE NETHERLANDS}

doi:10.1136/sextrans-2011-050108.102

R Heymans, S Bruisten, A Matser, T Heijman, H de Vries, M S van der Loeff. Health Service of Amsterdam, (GGD), Amsterdam, Netherlands

Background Sexual risk behaviour increased since the introduction of antiretroviral treatment against HIV-1. Consequently, since the 